\title{
MIMO-OFDM Channel Estimation in Presence of Carrier Frequency Offsets
}

\author{
Zhongshan Zhang, Wei Zhang and Chintha Tellambura \\ Department of Electrical and Computer Engineering \\ University of Alberta, Edmonton, Alberta, Canada T6G 2V4 \\ \{zszhang, wzhang, chintha\}@ece.ualberta.ca
}

\begin{abstract}
Optimal pilot design and placement for channel estimation in Multiple-input Multiple-output (MIMO) Orthogonal Frequency-Division Multiplexing (OFDM) systems in the presence of frequency offset are discussed. Both the singlefrequency-offset case and the multiple-frequency-offset case are treated. Constant-Envelope (CE) is shown to be a sufficient but not necessary condition for optimizing the mean square error (MSE). When the CE condition is relaxed, i.e., pilots with multiple envelope values, the power allocation and placement rules for the optimal MSE performance are derived. The Least-Squares (LS) and Linear Minimum Mean Square Error (LMMSE) estimators are designed for uncorrelated and correlated MIMO-OFDM channels, respectively. Both optimal adaptive pilot power allocation and suboptimal uniform pilot power allocation strategies are developed for the proposed LMMSE estimator. The adaptive allocation performs $4 \mathrm{~dB}$ better than the uniform allocation in the high noise region, but they both perform identically in the low noise region. Performance comparisons are made against several previous pilot designs due to [1]. The proposed LMMSE estimator significantly outperforms the LS estimator.
\end{abstract}

\section{INTRODUCTION}

Multiple-input Multiple-output (MIMO) technology is a popular method to increase the capacity of wireless systems [2]. However, at high data rates, MIMO channels become frequency-selective fading, which typically requires highcomplexity equalization and demodulation. This problem is typically overcome by using MIMO Orthogonal FrequencyDivision Multiplexing (MIMO-OFDM), which transforms a frequency-selective fading MIMO channel into a set of flatfading MIMO channels.

However, MIMO-OFDM systems are highly sensitive to frequency offsets, and these impair the accuracy of parameter estimation. For example, parameters estimation for MIMO flatfading channels is discussed in [3], where frequency offsets for different transmit and receive antenna pairs are assumed to be different. Another prevalent impairment is the channel estimation error, which can also degrade the MIMO-OFDM bit error rates. Optimal training signal design for frequencyselective block fading channel estimation in MIMO-OFDM systems is discussed in [4], which is based on the minimization of the MSE. Barhumi, Leus and Moonen (BLM) propose a high-quality channel estimator for a MIMO-OFDM channel based on frequency-domain uniformly placed pilots, and an optimal performance in terms of MSE can be achieved if the frequency offset and channel correlation are not considered [1]. MIMO-OFDM channel estimation for a correlated channel is discussed in [5], but without considering the effect of frequency offset. Training sequence design for MIMO channel estimation in the presence of a single frequency offset is discussed in [6], [7]. A robust optimal training signal design for MIMO-OFDM channel estimation while considering a single frequency offset and phase noise is proposed in [8]. Joint frequency offset and channel estimation with either single or multiple frequency offsets for MIMO frequency selective fading channels is discussed in [9], where pilots for different transmit antennas are orthogonal in the time-domain. Pilot optimization for MSE reduction is also studied in [9].

In this paper, optimal pilot design and placement for MIMOOFDM systems in the presence of multiple frequency offsets are developed. Just as in [3], the frequency offset of each transmit-receive antenna pair is assumed to be an Independent and Identically Distributed (i.i.d.) random variable (RV). Using the proposed pilots, the Least-Squares (LS) and Linear Minimum Mean Square Error (LMMSE) estimators are designed for uncorrelated and correlated MIMO-OFDM channels.

The remainder of this paper is organized as follows. The MIMO-OFDM system model is described in Section II, and the LS channel estimation in the presence of frequency offsets is analyzed in Section III. The optimal pilot design and placement for the LS channel estimator in the presence of a single or multiple frequency offsets are discussed in Section IV, and an LMMSE estimator for a correlated MIMO-OFDM channel is proposed in Section V. Numerical results are given in Section VI, followed by conclusions in Section VII.

Notation: $(\cdot)^{T},(\cdot)^{*}$ and $(\cdot)^{H}$ denote the transpose, complex conjugate and complex conjugate transpose. The imaginary unit is $\jmath=\sqrt{-1}$. A circularly symmetric complex Gaussian $\mathrm{RV} w$ with mean $m$ and variance $\sigma^{2}$ is denoted by $w \sim$ $\mathcal{C N}\left(m, \sigma^{2}\right) . \mathbf{I}_{N}$ is the $N \times N$ identity matrix, and $\mathbf{O}_{N}$ is the $N \times N$ all-zero matrix. $\mathbf{0}_{N}$ is the $N \times 1$ all-zero vector. $\mathbf{a}[i]$ is the $i$-th entry of vector $\mathbf{a}$, and $\|\mathbf{a}\|_{2}^{2}=\sum_{i}|\mathbf{a}[i]|^{2}$. $[\mathbf{B}]_{m n}$ is the $m n$-th entry of matrix $\mathbf{B}$. $(x)_{n}$ represents the remainder after division of $x$ by $n . \mathbb{E}\{X\}$ is the mean of $X$.

\section{MIMO-OFDM SIgNAL MODEL}

Input data bits of a MIMO-OFDM system are mapped to complex symbols drawn from a typical signal constellation, e.g., phase-shift keying (PSK) or quadrature amplitude modulation (QAM). An OFDM symbol is generated by taking the 
Inverse Discrete Fourier Transform (IDFT) of $N$ constellation symbols, where $N$ is the IDFT size, and the IDFT matrix $\mathbf{F}$ is defined as $[\mathbf{F}]_{n k}=\frac{1}{\sqrt{N}} e^{\frac{j 2 \pi n k}{N}}$ for $0 \leq n, k \leq N-1$. A cyclic prefix longer than the channel-response duration is used to mitigate Inter-Symbol-Interference (ISI). For a MIMO-OFDM system with $N_{t}$ transmit antennas and $N_{r}$ receive antennas, a $N \times 1$ vector $\mathbf{x}_{i}(z)$ is used to represent the $z$-th block of the frequency-domain symbols sent by the $i$-th transmit antenna, where $i \in\left\{1,2, \cdots, N_{t}\right\}$. In the following sections, when the discussion focuses on a single block, the temporal index $z$ will be omitted for brevity.

By using $h_{k, i}(n)$ to represent the discrete-time impulse response of the $n$-th tap channel between the $i$-th transmit and the $k$-th receive antennas, the related channel response vector can be represented as $\mathbf{h}_{k, i}=\left[\tilde{\mathbf{h}}_{k, i}^{T}, \mathbf{0}_{N-L_{\max }}^{T}\right]^{T}=$ $\left[h_{k, i}(0), h_{k, i}(1), \cdots, h_{k, i}\left(L_{\max }-1\right), \mathbf{0}_{N-L_{\max }}^{T}\right]^{T}$ with $L_{\max }$ representing the maximum length of all channels. We also use $\psi_{k, i}$ and $\varepsilon_{k, i}$ to represent the initial phase and the normalized frequency offset between the $i$-th transmit and the $k$-th receive antenna. Without loss of generality, we assume that $\psi_{k, i}$ for each $(k, i)$ has been estimated and compensated for and that, therefore, $\psi_{k, i}=0$. In this paper, both the single frequency offset (SFO) case and the multiple frequency offsets (MFO) case are analyzed. For the SFO case, $\varepsilon_{k, i}=\varepsilon, \forall k, \forall i$, where $\varepsilon$ is an RV with mean zero and variance $\sigma_{\epsilon}^{2}$; and for the MFO case, $\varepsilon_{k, i}$ for each $(k, i)$ is assumed to be an i.i.d. RV with mean zero and variance $\sigma_{\epsilon}^{2}$. In both cases, the frequency offsets vary from one symbol to the next. The $q$-th received vector sample is represented as $\mathbf{y}(q)=\left[\mathbf{y}_{1}^{T}(q), \mathbf{y}_{2}^{T}(q), \cdots, \mathbf{y}_{N_{r}}^{T}(q)\right]^{T}$, where $\mathbf{y}_{k}(q)=\sum_{i=1}^{N_{t}} \mathbf{E}_{k, i} \mathbf{F H}_{k, i} \mathbf{x}_{i}(q)+\mathbf{w}_{k}(q), \mathbf{E}_{k, i}=$ $\operatorname{diag}\left\{1, e^{\frac{j 2 \pi \varepsilon_{k, i}}{N}}, \cdots, e^{\frac{j 2 \pi \varepsilon_{k, i}(N-1)}{N}}\right\}$, and $\mathbf{w}_{k}(q)$ is a vector of additive white Gaussian noise (AWGN) with $\mathbf{w}_{k}(q)[n] \sim$ $\mathcal{C N}\left(0, \sigma_{w}^{2}\right) . \mathbf{X}_{i}(q)=\mathbf{X}_{i}^{d}(q)+\mathbf{X}_{i}^{p}(q)=\operatorname{diag}\left\{\mathbf{x}_{i}^{d}(q)\right\}+$ $\operatorname{diag}\left\{\mathbf{x}_{i}^{p}(q)\right\}$ with $\mathbf{x}_{i}^{d}(q)$ and $\mathbf{x}_{i}^{p}(q)$ representing some $N \times 1$ data and pilot vector, respectively. $\mathbf{F}_{\left(L_{\max }\right)}$ here is the first $L_{\max }$ rows of $\mathbf{F}$.

At the $k$-th receive antenna, by taking the Discrete Fourier Transform (DFT) to the received vector, we obtain

$$
\begin{aligned}
\mathbf{r}_{k}(q) & =\mathbf{F}^{H} \mathbf{y}_{k}(q)=\sum_{i=1}^{N_{t}} \underbrace{\sqrt{N} \mathbf{E}_{k, i}^{\mathrm{cir}} \mathbf{X}_{i}^{p}(q) \mathbf{F}_{\left(L_{\max }\right)}^{H}}_{\mathbf{P}_{k, i}(q)} \tilde{\mathbf{h}}_{k, i}) \\
& +\sum_{i=1}^{N_{t}} \underbrace{\sqrt{N} \mathbf{E}_{k, i}^{\mathrm{cir}} \mathbf{X}_{i}^{d}(q) \mathbf{F}_{\left(L_{\max }\right)}^{H}}_{\mathbf{D}_{k, i}(q)\left(N \times L_{\max }\right)} \tilde{\mathbf{h}}_{k, i}+\underbrace{\mathbf{F}^{H} \mathbf{w}_{k}(q)}_{\boldsymbol{\eta}_{k}(q)(N \times 1)},
\end{aligned}
$$

where $\mathbf{E}_{k, i}^{\mathrm{cir}}=\mathbf{F}^{H} \mathbf{E}_{k, i} \mathbf{F}$. In the following sections, $E_{p}$ is used to represent the total pilot power. Assuming that the pilots are modulated into consecutive $M$ symbols $(M \geq 1)$, the received vector becomes

$$
\mathbf{r}_{k}=\left[\mathbf{r}_{k}^{T}(0), \cdots, \mathbf{r}_{k}^{T}(M-1)\right]^{T}=\mathbf{P}_{k} \mathbf{h}_{k}+\mathbf{D}_{k} \mathbf{h}_{k}+\boldsymbol{\eta}_{k},
$$

where $\mathbf{P}_{k}=\left[\begin{array}{ccc}\mathbf{P}_{k, 1}(0) & \ldots & \mathbf{P}_{k, N_{t}}(0) \\ \vdots & \ddots & \vdots \\ \mathbf{P}_{k, 1}(M-1) & \ldots & \mathbf{P}_{k, N_{t}}(M-1)\end{array}\right]$,
$\mathbf{D}_{k}=\left[\begin{array}{ccc}\mathbf{D}_{k, 1}(0) & \ldots & \mathbf{D}_{k, N_{t}}(0) \\ \vdots & \ddots & \vdots \\ \mathbf{D}_{k, 1}(M-1) & \ldots & \mathbf{D}_{k, N_{t}}(M-1)\end{array}\right], \mathbf{h}_{k}=$
$\left[\tilde{\mathbf{h}}_{k, 1}^{T}, \cdots, \tilde{\mathbf{h}}_{k, N_{t}}^{T}\right]^{T}$ and $\boldsymbol{\eta}_{k}=\left[\boldsymbol{\eta}_{k}^{T}(0), \cdots, \boldsymbol{\eta}_{k}^{T}(M-1)\right]^{T}$.

III. LS FREQUENCY OFFSET AND CHANNEL ESTIMATION IN MIMO-OFDM SYSTEMS

In Sections III and IV, no channel correlation is considered. The correlated case will be treated in Section V. For LS channel estimation, $\mathbf{P}_{k}$ should be of full-column rank, so that $M N \geq L_{\max } N_{t}$. By defining the pseudo-inverse of $\mathbf{P}_{k}$ as $\mathbf{P}_{k}^{\dagger}=\left(\mathbf{P}_{k}^{H} \mathbf{P}_{k}\right)^{-1} \mathbf{P}_{k}^{H}$, the LS estimation of $\mathbf{h}_{k}$ is given by

$$
\hat{\mathbf{h}}_{k \mid \varepsilon_{k, 1}, \cdots, \varepsilon_{k, N_{t}}}=\mathbf{P}_{k}^{\dagger} \mathbf{r}_{k}=\mathbf{h}_{k}+\mathbf{P}_{k}^{\dagger} \mathbf{D}_{k} \mathbf{h}_{k}+\mathbf{P}_{k}^{\dagger} \boldsymbol{\eta}_{k} .
$$

The MSE of the LS estimator $\hat{\mathbf{h}}_{k \mid \varepsilon_{k, 1}, \cdots, \varepsilon_{k, N_{t}}}$ is given by

$$
\begin{aligned}
& \operatorname{MSE}\left(\hat{\mathbf{h}}_{k \mid \mathrm{LS}}\right)=\frac{1}{L_{\text {max }} N_{t}} \mathbb{E}\left\{\left\|\hat{\mathbf{h}}_{k \mid \varepsilon_{k, 1}, \cdots, \varepsilon_{k, N_{t}}}-\mathbf{h}_{k}\right\|_{2}^{2}\right\} \\
& =\frac{\operatorname{trace}\left\{\mathbf{V}_{k}^{H}\left(\mathbf{P}_{k}^{H} \mathbf{P}_{k}\right)^{-2} \mathbf{V}_{k} \mathbf{\Phi}_{k}\right\}}{L_{\text {max }} N_{t}}+\frac{\sigma_{w}^{2} \operatorname{trace}\left\{\left(\mathbf{P}_{k}^{H} \mathbf{P}_{k}\right)^{-1}\right\}}{L_{\max } N_{t}}
\end{aligned}
$$

where $\boldsymbol{\Phi}_{k}=\mathbb{E}\left\{\mathbf{h}_{k} \mathbf{h}_{k}^{H}\right\}, \mathbf{V}_{k}=\mathbf{P}_{k}^{H} \mathbf{D}_{k}$, and $\mathbf{P}_{k}^{H} \mathbf{P}_{k}$ can be represented as

$$
\mathbf{P}_{k}^{H} \mathbf{P}_{k}=\left[\begin{array}{ccc}
\mathbf{G}_{k, 1,1} & \ldots & \mathbf{G}_{k, 1, N_{t}} \\
\vdots & \ddots & \vdots \\
\mathbf{G}_{k, N_{t}, 1} & \ldots & \mathbf{G}_{k, N_{t}, N_{t}}
\end{array}\right]
$$

where

$$
\mathbf{G}_{k, m, n}=N \sum_{i=0}^{M-1} \mathbf{F}_{\left(L_{\max }\right)} \mathbf{X}_{m}^{p H}(i) \mathbf{E}_{k, m, n}^{\mathrm{cir}} \mathbf{X}_{n}^{p}(i) \mathbf{F}_{\left(L_{\max }\right)}^{H}
$$

with $\mathbf{E}_{k, m, n}^{\mathrm{cir}}=\mathbf{F}^{H} \mathbf{E}_{k, n} \mathbf{E}_{k, m}^{H} \mathbf{F}$.

\section{Optimal Pilot Design and Placement for the LS CHANNEL ESTIMATION}

In the presence of frequency offsets, MSE $\left(\hat{\mathbf{h}}_{k \mid \mathrm{LS}}\right)$ can be minimized if the eigenvalues of $\mathbf{P}_{k}^{H} \mathbf{P}_{k}$ are identical. This condition requires the following:

\section{Proposition 1:}
1. $\mathbf{G}_{k, n, n}=\frac{E_{p}}{N_{t}} \mathbf{I}_{L_{\max }}, 1 \leq n \leq N_{t}$.
2. $\mathbf{G}_{k, m, n \neq m}=\mathbf{O}_{L_{\text {max }}}$ for each $(m, n \neq m)$.

In order to find the pilots and their placements that satisfy Proposition 1, we first assume that a total of $\mathcal{N}_{p}$ pilots are allocated to each transmit antenna, and that the frequencydomain indexes of the pilots for the $n$-th transmit antenna are $\left(\theta_{n, 1}, \cdots, \theta_{n, \mathcal{N}_{p}}\right)$, where $0 \leq \theta_{n, 1}<\cdots<\theta_{n, \mathcal{N}_{p}} \leq N-1$. In the following sections, pilot subcarriers of either orthogonal (in either the time-domain or the frequency-domain) or nonorthogonal (pilot subcarriers for the different transmit antennas 
are overlap) will be discussed. Let us analyze the optimal pilot design and placement in two cases; i.e., SFO and MFO:

\section{A. SFO Case}

In the SFO case, when pilot subcarriers for the different transmit antennas are orthogonal in the frequency-domain, $\mathbf{G}_{k, m, n \neq m}=\mathbf{O}_{L_{\max }}$ for each $(m, n \neq m)$ is satisfied. When overlapped pilot subcarriers are allocated to each transmit antenna,

$$
\sum_{z=1}^{\mathcal{N}_{p}}\left[\sum_{i=0}^{M-1} \mathbf{X}_{m}^{p H}(i) \mathbf{X}_{n}^{p}(i)\right]_{\theta_{z} \theta_{z}} e^{\frac{j 2 \pi \theta_{z}(l-s)}{N}}=0
$$

should be satisfied for each $0 \leq l, s \leq L_{\max }-1$ to make $\mathbf{G}_{k, m, n \neq m}=\mathbf{O}_{L_{\max }}$. The solution of (7) is found to be

$$
\begin{aligned}
\arg \left\{\left[\mathbf{X}_{m}^{p}(i)\right]_{\theta_{z} \theta_{z}}\right\}=\frac{2 \pi \theta_{z} K_{p} m}{N}, & \\
\text { s.t. } \quad & L_{\max } \leq \mathcal{N}_{p} \leq N, \frac{N}{\mathcal{N}_{p}}=\text { integer; } \\
& K_{p} \geq L_{\text {max }} ; \frac{\theta_{z} K_{p}(n-m)}{N} \neq \text { integer; } \\
& \left(\theta_{2} \cdot l-\theta_{1} \cdot l\right)_{N}=\cdots=\left(\theta_{1} \cdot l-\theta_{\mathcal{N}_{p}} \cdot l\right)_{N} ; \\
& \sum_{i=1}^{M}\left|\left[\mathbf{X}_{m}^{p}(i)\right]_{\theta_{z} \theta_{z}}\right|^{2}=\frac{E_{p}}{\mathcal{N}_{p} N_{t}} ; \\
& \sum_{i=1}^{M} \sum_{z=1}^{\mathcal{N}_{p}}\left|\left[\mathbf{X}_{m}^{p}(i)\right]_{\theta_{z} \theta_{z}}\right|^{2}=\frac{E_{p}}{N_{t}},
\end{aligned}
$$

for $z=1,2, \cdots, \mathcal{N}_{p}, m=1,2, \cdots, N_{t}, i=0,1, \cdots, M-1$.

\section{B. MFO Case}

In the MFO case, when the pilot subcarriers for the different transmit antennas are orthogonal in the frequency-domain, $\mathbf{G}_{k, m, n \neq m}=\mathbf{O}_{L_{\max }}$ is achieved for each $m \neq n$, and, therefore, all pilots satisfy (8) are the optimal pilots in terms of MSE. When $\theta_{m, z}=\theta_{n \neq m, z}=\theta_{z}$ for each $1 \leq m, n \neq$ $m \leq N_{t}$ and $z=1,2, \cdots \mathcal{N}_{p}$, the pilot design is analyzed in the following two cases:

1) $M \geq N_{t}$ : In this case, $\mathbf{G}_{k, m, n}=\mathbf{O}_{L_{\text {max }}}$ is achieved if the pilots transmitted by the different transmit antennas are orthogonal in the time-domain, i.e.,

$$
\begin{aligned}
& {\left[\mathbf{X}_{m}^{p}(i)\right]_{\theta_{z} \theta_{z}} \neq 0 \Rightarrow\left[\mathbf{X}_{n \neq m}^{p}(i)\right]_{\theta_{z} \theta_{z}}=0} \\
& {\left[\mathbf{X}_{m}^{p}(i)\right]_{\theta_{z} \theta_{z}} \neq 0 \Rightarrow\left[\mathbf{X}_{m}^{p}(i)\right]_{\left(\theta_{z}+\frac{N}{2}\right)_{N}\left(\theta_{z}+\frac{N}{2}\right)_{N}} \neq 0}
\end{aligned}
$$

for each $1 \leq m, n \neq m \leq N_{t}$.

2) $M \leq N_{t}$ : In this case, pilots transmitted by the different transmit antennas cannot be orthogonal in the time-domain. Since the Inter-Pilot-Interference (IPI) cannot be totally eliminated, we can only get the suboptimal pilots in terms of the $\mathrm{MSE}$, as given by

$$
\begin{array}{r}
\mathbf{P}_{k}^{\text {subopt }}=\arg \min _{\mathbf{P}_{k}} \mathbb{E}\left\{\operatorname{MSE}\left(\hat{\mathbf{h}}_{k \mid \varepsilon_{k, 1}, \cdots, \varepsilon_{k, N_{t}}}\right)\right\}, \\
\text { s.t. } \operatorname{trace}\left\{\left(\mathbf{P}_{k}^{H} \mathbf{P}_{k}\right)\right\}=L_{\text {max }} E_{p} .
\end{array}
$$

To resolve (10),

$$
\sum_{m=1}^{N_{t}} \sum_{i=0}^{M-1} \mathbf{F}_{\left(L_{\max }\right)} \mathbf{X}_{m}^{p H}(i) \mathbf{F}^{H}=\frac{E_{p}}{N_{t}} \underbrace{[\mathbf{B}, \cdots, \mathbf{B}]}_{L_{\max } \times N}
$$

should be satisfied, where $\mathbf{B}=$
$[\underbrace{\mathbf{I}_{L_{\max }}, \cdots, \mathbf{I}_{L_{\max }}}_{L_{\max } \times L_{\max } N_{t}}, \mathbf{O}_{L_{\max } \times\left(\mathcal{N}_{p}-L_{\max } N_{t}\right)}]$, which requires suboptimal pilots as

$$
\begin{aligned}
& \arg \left\{\left[\mathbf{X}_{m}^{p}(i)\right]_{\theta_{z} \theta_{z}}\right\}=\frac{2 \pi \theta_{z}(m-1) L_{\max }}{N}, \\
& \text { s.t. } \quad L_{\max } N_{t} \leq \mathcal{N}_{p} \leq N, \frac{N}{\mathcal{N}_{p}}=\text { integer; } \\
& \left(\theta_{2} \cdot l-\theta_{1} \cdot l\right)_{N}=\cdots=\left(\theta_{1} \cdot l-\theta_{\mathcal{N}_{p}} \cdot l\right)_{N} ; \\
& \frac{\theta_{z}(n-m) L_{\max }}{N} \neq \text { integer; } \\
& {\left[\mathbf{X}_{m}^{p}(i)\right]_{\theta_{1} \theta_{1}}=\cdots=\left[\mathbf{X}_{m}^{p}(i)\right]_{\theta_{\mathcal{N}_{p}} \theta_{\mathcal{N}_{p}}} \geq 0 ;} \\
& \sum_{i=1}^{M}\left|\left[\mathbf{X}_{m}^{p}(i)\right]_{\theta_{z} \theta_{z}}\right|^{2}=\frac{E_{p}}{\mathcal{N}_{p} N_{t}} ; \\
& \sum_{i=1}^{M} \sum_{z=1}^{\mathcal{N}_{p}}\left|\left[\mathbf{X}_{m}^{p}(i)\right]_{\theta_{z} \theta_{z}}\right|^{2}=\frac{E_{p}}{N_{t}} .
\end{aligned}
$$

Here we should note that both the optimal pilots and the suboptimal pilots are uniformly placed in the frequencydomain, and may suffer a performance degrade due to the pilots losses at the virtual subcarriers. One way to mitigate this performance loss here is to make the pilot distance larger than the virtual-subcarrier bandwidth and to guarantee that $\mathcal{N}_{p} \geq L_{\max } N_{t}$ simultaneously.

\section{Linear Minimum Mean SQuare ERror (LMMSE) ESTIMATOR USING CHANNEL CORRELATION}

Sections III to IV considered pilot design in an uncorrelated MIMO-OFDM channel. However, channel correlation can be exploited to improve the performance of channel estimation [5].

Define

$$
\mathbf{r}=\left[\mathbf{r}_{1}^{T}, \cdots, \mathbf{r}_{N_{r}}^{T}\right]^{T}=\mathbf{P h}+\mathbf{D h}+\boldsymbol{\eta},
$$

where $\mathbf{P}=\operatorname{diag}\left\{\mathbf{P}_{1}, \cdots, \mathbf{P}_{N_{r}}\right\}, \mathbf{D}=\operatorname{diag}\left\{\mathbf{D}_{1}, \cdots, \mathbf{D}_{N_{r}}\right\}$, $\mathbf{h}=\left[\mathbf{h}_{1}^{T}, \cdots, \mathbf{h}_{N_{r}}^{T}\right]^{T}$ and $\boldsymbol{\eta}=\left[\boldsymbol{\eta}_{1}^{T}, \cdots, \boldsymbol{\eta}_{N_{r}}^{T}\right]^{T}$. Also define $\boldsymbol{\Omega}=\left(\mathbf{I}_{L_{\max } N_{t} N_{r}}+\left(\mathbf{P}^{H} \mathbf{P}\right)^{-1} \mathbf{P}^{H} \mathbf{D}\right)$, and $\boldsymbol{\Phi}=\mathbb{E}\left\{\mathbf{h} \mathbf{h}^{H}\right\}$, an LMMSE estimator can be designed as

$$
\hat{\mathbf{h}}_{\mathrm{LMMSE}}=\left(\sigma_{w}^{2} \boldsymbol{\Phi}^{-1}+\boldsymbol{\Omega}^{H}\left(\mathbf{P}^{H} \mathbf{P}\right) \boldsymbol{\Omega}\right)^{-1} \boldsymbol{\Omega}^{H} \mathbf{P}^{H} \mathbf{r} .
$$

The MSE of (14) is given by

$$
\operatorname{MSE}\left(\hat{\mathbf{h}}_{\mathrm{LMMSE}}\right)=\frac{\operatorname{trace}\left\{\mathbf{C}_{\mathbf{e}}\right\}}{L_{\max } N_{t} N_{r}},
$$


where $\mathbf{C}_{\mathbf{e}}=\mathbb{E}\left\{\left(\hat{\mathbf{h}}_{\text {LMMSE }}-\mathbf{h}\right)\left(\hat{\mathbf{h}}_{\text {LMMSE }}-\mathbf{h}\right)^{H}\right\}$.

Using the eigenvalue decomposition of $\mathbf{C}_{\mathbf{e}}$, we have

$$
\begin{aligned}
\operatorname{MSE}\left(\hat{\mathbf{h}}_{\text {LMMSE }}\right)=\frac{\operatorname{trace}\left\{\mathbf{U C}_{\mathrm{e}} \mathbf{U}^{H}\right\}}{L_{\max } N_{t} N_{r}} \\
=\frac{\operatorname{trace}\left\{\left(\boldsymbol{\Lambda}_{\phi}^{-1}+\frac{1}{\sigma_{w}^{2}} \mathbf{U}^{H} \boldsymbol{\Omega}^{H}\left(\mathbf{P}^{H} \mathbf{P}\right) \boldsymbol{\Omega} \mathbf{U}\right)^{-1}\right\}}{L_{\text {max }} N_{t} N_{r}},
\end{aligned}
$$

where $\mathbf{U}$ is a $L_{\max } N_{t} N_{r} \times L_{\max } N_{t} N_{r}$ unitary matrix,

$$
\boldsymbol{\Lambda}_{\phi}=\mathbf{U} \boldsymbol{\Phi} \mathbf{U}^{H}=\operatorname{diag}\left\{\lambda_{\phi, 0}^{2}, \cdots, \lambda_{\phi, L_{\max } N_{t} N_{r}-1}^{2}\right\}
$$

with $\lambda_{\phi, n}^{2}$ representing the $n$-th eigenvalue of $\boldsymbol{\Phi}$. Let $\lambda_{p, n}^{2}$ be the $n$-th eigenvalue of $\mathbf{P}^{H} \mathbf{P}$. The optimal set of eigenvalues that minimize MSE $\left(\hat{\mathbf{h}}_{\mathrm{LMMSE}}\right)$ is given by

$$
\lambda_{p, n}^{2}=\frac{\left(N_{r} L_{\max } E_{p}+\sum_{m=0}^{L_{\max } N_{t} N_{r}-1} \frac{\sigma_{w}^{2}}{\lambda_{\phi, m}^{2}}\right)}{L_{\max } N_{t} N_{r}-1}-\frac{\sigma_{w}^{2}}{\lambda_{\phi, n}^{2}}
$$

for each $0 \leq n \leq L_{\max } N_{t} N_{r}-1$.

\section{NUMERICAL RESULTS}

Our simulations assume that $N=128$ and $L_{\max }=4$, and that each tap of the wireless channel is a complex Gaussian $\mathrm{RV}$ with mean zero and variance $\sigma_{\epsilon}^{2}$. The Normalized-Pilotto-Noise Ratio (NPNR), i.e., NPNR $=\frac{E_{p}}{N_{t} \mathcal{N}_{p} \sigma_{w}^{2}}$, is used to represent the normalized Signal-to-Noise Ratio (SNR) of each pilot subcarrier for each transmit antenna. Without loss of generality, the average power of each pilot subcarrier is assumed to be identical to that of each data subcarrier. Each frequency offset is assumed to be an i.i.d. Gaussian RV with zero mean and variance $\sigma_{\varepsilon}^{2}$.

Fig. 1 to Fig. 2 illustrate the performance comparison between the proposed LS estimator and the BLM estimator in the presence of frequency offsets, for $M=1$ and $N_{t}=4$. In the SFO case, a performance floor will always appear in the BLM estimator at a high NPNR, and a larger $\sigma_{\varepsilon}^{2}$ implies a higher MSE. This performance floor can be eliminated by the proposed estimator. A performance floor will appear in both estimators in the MFO case, because the IPI cannot be totally eliminated. However, the proposed estimator still outperforms the BLM estimator. For example, for an overlapped pilot placement, when NPNR $=20 \mathrm{~dB}$, the MSE of the proposed estimator is about $1.6 \times 10^{-3}$ (or $2.3 \times 10^{-3}$ ) with $\sigma_{\varepsilon}^{2}=10^{-3}$ (or $\sigma_{\varepsilon}^{2}=10^{-2}$ ), and that of the BLM estimator is about $5.5 \times 10^{-3}$ (or $3.6 \times 10^{-2}$ ) with $\sigma_{\varepsilon}^{2}=10^{-3}\left(\right.$ or $\sigma_{\varepsilon}^{2}=10^{-2}$ ), as shown in Fig. 1.

When $N_{t}>1$, pilots for each transmit antenna can be modulated into consecutive $M>1$ symbols, and the envelope for each subcarrier of each symbol can be either a CE or not. The simulation results with $N_{t}=M=4$ are illustrated in Fig. 3, where frequency-domain orthogonal pilot placement is assumed for the different transmit antennas in each symbol. The proposed pilots, either a CE or Multiple-Envelope, can achieve the same performance advantage over that of the BLM estimator, provided that the total pilots power $E_{p}$ remains fixed. For a given $E_{p}$, an identical performance can be achieved in the proposed estimator with either $M=1$ or $M>$ 1. For example, when $N_{t}=4, \sigma_{\varepsilon}^{2}=10^{-2}$ and NPNR $=20 \mathrm{~dB}$, an MSE of about $1.5 \times 10^{-2}$ can be achieved with either $M=$ 1 or $M=4$, as shown in Fig. 2 and Fig. 3. No matter what pilots are used, the pilots over consecutive multiple symbols have no performance advantage over that of single-symbol pilots in terms of the MSE, and more seriously, multiplesymbol pilots decrease the spectrum efficiency. Nonetheless, multiple-symbol pilot modulation is applied for two main reasons: (1) multiple-symbol pilot modulation reduces the peak power of each pilot subcarrier in each symbol; and (2) multiple-symbol pilot modulation has an advantage over single-symbol pilot modulation in tracking the time-variant channel.

When the covariance matrix of the wireless channel is available at both the transmitter and the receiver, the LMMSE estimator can be used to improve the estimation accuracy. Fig. 4 compares the channel estimation performance between the proposed LS and the proposed LMMSE estimators. Multiple frequency offsets are considered in this simulation with $\sigma_{\varepsilon}^{2}=10^{-2}$. In comparison to the LS estimator, the LMMSE estimator with CE pilots can considerably reduce the MSE, and this lower MSE is achieved by applying adaptive pilot power allocation at the transmitter. For example, when $N_{t}=$ $N_{r}=2$, the performance advantage of the proposed LMMSE estimator with adaptive pilot power allocation over that with CE pilots at a low NPNR is about $3.1 \mathrm{~dB}$, and its performance advantage over that of the LS estimator is about $5.8 \mathrm{~dB}$.

\section{CONCLUSIONS}

The optimal pilot design and placement for channel estimation in MIMO-OFDM with multiple frequency offsets were discussed. The IPI was eliminated in the proposed estimator, and, therefore, a performance advantage over that of conventional estimators was achieved. Given a total pilot power $E_{p}$, the pilots for the different transmit antennas was modulated into one or consecutive multiple OFDM symbols with each pilot subcarrier in each symbol being modulated as CE or not (CE pilots are required only when $M=1$ ). With the channel covariance matrix known at both the transmitter and the receiver, an LMMSE estimator with CE pilots modulation was designed based on the proposed optimal pilots. It outperforms the proposed LS estimator in terms of the MSE. Moreover, by using adaptive pilot power allocation at the transmitter, it achieved a much lower MSE than that of CE pilot modulation.

\section{REFERENCES}

[1] I. Barhumi, G. Leus, and M. Moonen, "Optimal training design for MIMO OFDM systems in mobile wireless channels," IEEE Trans. Signal Processing, vol. 51, no. 6, pp. 1615-1624, June 2003.

[2] G. J. Foschini, "Layered space-time architecture for wireless communication in a fading environment when using multi-element antennas," Bell Labs Tech. J.,, vol. 1, pp. 41-59, 1996. 


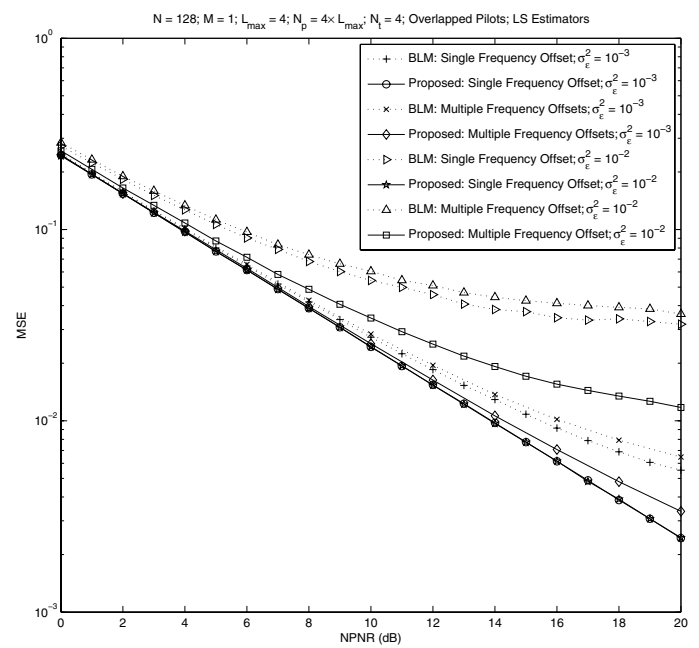

Fig. 1. LS channel estimation in MIMO-OFDM systems with $M=1$, $N_{t}=4$ and pilot subcarriers of different transmit antennas being overlapped.

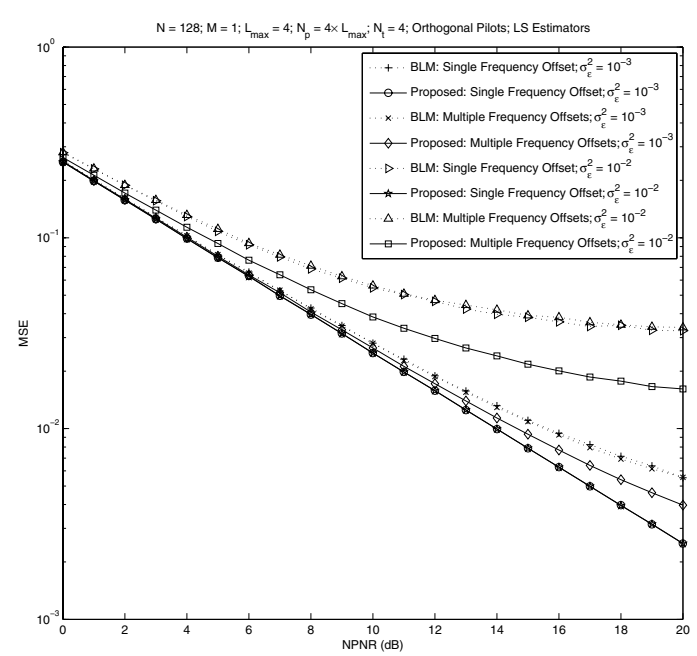

Fig. 2. LS channel estimation in MIMO-OFDM systems with $M=1$, $N_{t}=4$ and pilot subcarriers of different transmit antennas being orthogonal

[3] O. Besson and P. Stoica, "On parameter estimation of MIMO flat-fading channels with frequency offsets," IEEE Trans. Signal Processing, vol. 51, no. 3, pp. 602-613, Mar. 2003.

[4] H. Minn and N. Al-Dhahir, "Optimal training signals for MIMO OFDM channel estimation," IEEE Trans. Wireless Commun., vol. 5, no. 5, pp. 1158-1168, May 2006.

[5] H. Zhang, Y. Li, A. Reid, and J. Terry, "Channel estimation for MIMO OFDM in correlated fading channels," in Proc. IEEE Int. Conf. Communications (ICC), vol. 4, May 2005, pp. 2626-2630.

[6] H. Minn and N. Al-Dhahir, "MIMO-OFDM training signal design in the presence of frequency offset and phase noise," in Information, Communications and Signal Processing, 2005 Fifth International Conference on, Dec. 2005, pp. 263-267.

[7] M. Ghogho and A. Swami, "Training design for multipath channel

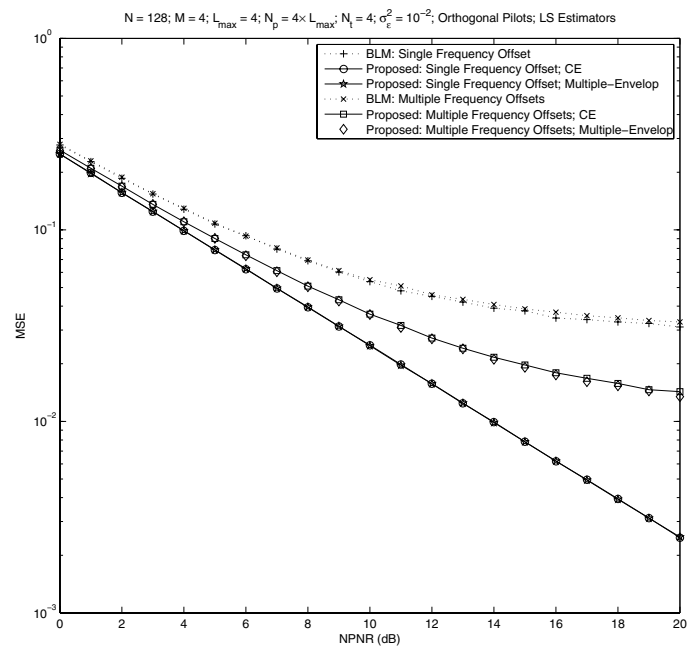

Fig. 3. LS channel estimation in MIMO-OFDM systems with $M=4$, $N_{t}=4$ and pilot subcarriers of different transmit antennas being orthogonal.

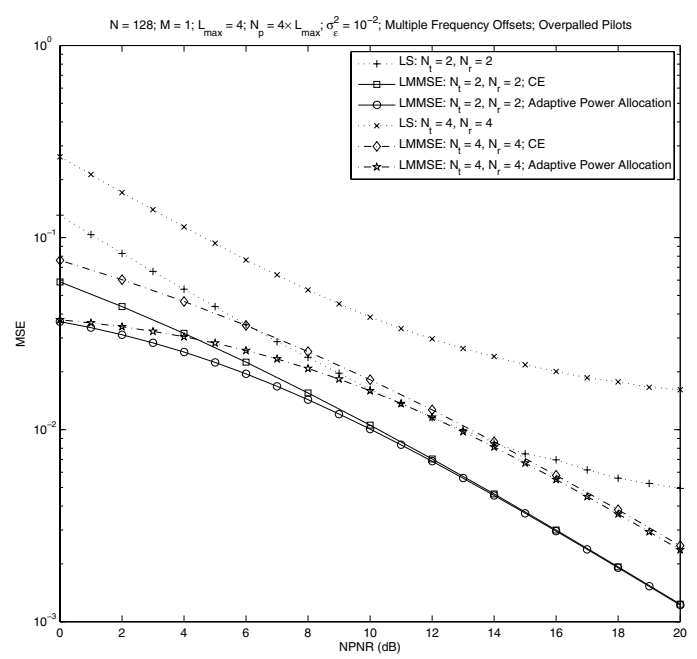

Fig. 4. Performance comparison between LS and LMMSE estimators.

and frequency-offset estimation in MIMO systems," IEEE Trans. Signal Processing, vol. 54, no. 10, pp. 3957-3965, Oct. 2006.

[8] H. Minn, N. Al-Dhahir, and Y. Li, "Optimal training signals for MIMO OFDM channel estimation in the presnece of frequency offset and phase noise," IEEE Trans. Commun., vol. 54, no. 6, pp. 1081-1096, June 2006.

[9] T. Cui and C. Tellambura, "Channel and frequency offset estimation and training sequence design for MIMO frequency selective channels," IEEE Trans. Wireless Commun., Submitted to appear. 